Journal of Guidance, Control, and Dynamics, Volume 42, Issue 4, 2018, pp. 941-948

DOI:10.2514/1.G003971

\title{
Three-Dimensional Optimal Impact Time Guidance for Anti-Ship Missiles
}

\author{
Shaoming He* \\ Cranfield University, Cranfield MK43 OAL, UK \\ Defu Lin ${ }^{\dagger}$ \\ Beijing Institute of Technology, Beijing 100081, P.R. China
}

\section{Introduction}

The primary objective of missile guidance laws is to drive the missile to intercept a specific target with zero miss distance. Proportional navigation guidance (PNG) has been proved to be an efficient and simple guidance algorithm for missile systems, thus showing wide applications in the past few decades [1]. The optimality of PNG was analyzed in [2] and its extension to three-dimensional (3D) scenario can be found at [3]. In the context of modern warfare, many high-value battleships, like destroyers and aircraft carriers, are equipped with powerful self-defense systems against anti-ship missiles [4]. In order to penetrate these formidable defensive systems, the concept of salvo attack or simultaneous attack was introduced: many missiles are required to hit a battleship simultaneously, albeit their different initial locations. One typical solution of simultaneous attack is impact time control guidance. Generally, impact time control can be classified into two categories: (1) specify the desired impact time and control each missile to satisfy the desired impact time constraint individually; and (2) synchronize the impact time either in a distributed or decentralized fashion through a communication network among all interceptors.

By using linearized kinematics with optimal control theory, the authors in [4] proposed an impact time control guidance law. The resulting command was proved to be a PNG term in conjunction with a feedback term for regulating the impact time error. This work seems to be the first paradigm in the area of salvo attack. A similar idea was utilized in [5] to extend [4] to satisfy both impact angle and impact time constraints. In this reference, the jerk command was chosen as the control input to provide one additional degree of freedom for impact time control. The authors in [6] further suggested an impact time guidance based on nonlinear kinematics. The resulting guidance command shows similar form as [4], thus justifying the applicability of [4] in reality. A biased PNG for impact time control was introduced in [7], where the estimated time-to-go was based on Gaussian hypergeometric and beta function. Using optimal error dynamics, the authors in [8] suggested a generalized optimal guidance law for impact time control. A terminal guidance law with numerical time-to-go estimation for hypersonic vehicle was proposed in [9] to cater for the impact time constraint.

\footnotetext{
*PhD Candidate, School of Aerospace, Transport and Manufacturing, College Road, AIAA Student Member, Email: shaoming.he.cn@gmail.com

†Professor, School of Aerospace Engineering, 5th Zhongguancun South Street, P.O. Box 327, Email: uavi@bit.edu.cn
} 
Apart from biased PNG concept, impact time control was also studied in the context of nonlinear control theory. With dedicated Lyapunov functions, nonlinear impact time control guidance laws were developed in [10, 11]. The singularity issue associated with the guidance command was also rigorously analyzed in [10]. Considering seeker's field-of-view limit, a terminal sliding mode guidance law was formulated in [12] to achieve the objective of salvo attack. Using PNG-based time-to-go estimations, sliding mode control was leveraged in [13-15] to reduce the impact time error. Through line-of-sight (LOS) shaping, impact time constraint was satisfied in [16] by using sliding mode control to drive the system to converge to the desired polynomial error dynamics. Different from [16], the concept of look angle shaping was leveraged to cater for impact time control in [17-20]. Combing sliding mode control with the virtual target concept, a impact time and impact angle constraint guidance law was proposed in [21]. A new impact time and impact angle control trajectory shaping guidance was suggested in [22] based on the elliptic geometric rule.

By sharing the information on predicted time-to-go among all missiles, a cooperative guidance law was suggested in [23] for simultaneous attack. A general solution to cooperative guidance problem was provided in [24] based on coordination algorithms. Following the concept of two-stage guidance, a guidance law to achieve simultaneous attack of multiple missiles was proposed in [25], where a linear distributed consensus protocol was suggested in the first stage to generate favored initial conditions for the latter stage.

Note that most previous impact time control guidance laws are dedicated for two-dimensional (2D) engagement scenarios, thus ignoring the cross couplings between horizontal and vertical channels. It is well known that the application of 2D guidance laws in realistic 3D engagements is valid for roll-stabilized interceptors. However, designing 3D guidance law is meaningful since it can fully exploit the synergism effect of horizontal and vertical planes. Therefore, 3D guidance law is more beneficial if the effect of cross couplings cannot be neglected. Although the Lyapunov-based guidance law [10] and the consensus-based guidance law [25] are directly derived using 3D kinematics, they are limited to address the optimality issue because they were formulated based on nonlinear control approaches rather than the optimal control framework. Furthermore, these two guidance laws only guarantee asymptotical convergence as proved by the authors.

Motivated by the aforementioned observations, this Note aims to propose a generalized optimal 3D guidance law for anti-ship missiles to satisfy the impact time constraint. For this purpose, we utilize a composite guidance command, similar to [4, 6, 8], consisting of an optimal 3D PNG part and a feedback loop for regulating the impact time error. In determining the error feedback term, we first generalize the 2D PNG-based time-to-go estimation approach [6] to the 3D homing case. In order to drive the predicted time-to-go to its desired pattern, the optimal error dynamics method, developed in [8], was utilized to design the error feedback command. The finite-time convergence of the impact time error and the optimality of the proposed guidance law are also analyzed. Notice that the guidance algorithm developed can be viewed as an extension of [8] to a realistic 3D scenario with more rigorous analysis about guidance command and guidance gain selection. Compared with the 2D optimal impact time guidance law [8], the proposed guidance law 


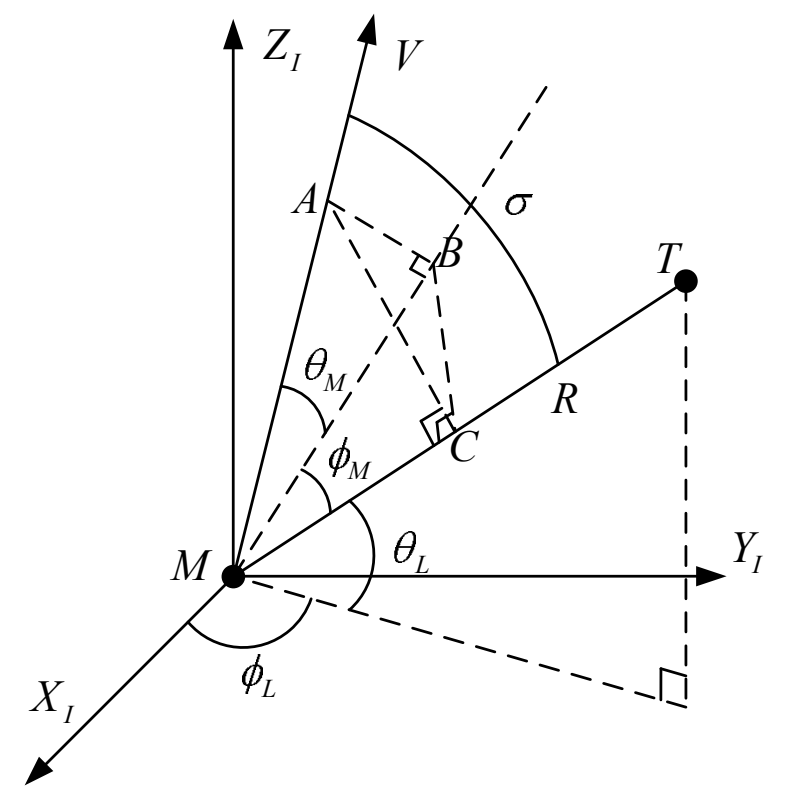

Fig. 1 Three-dimensional homing engagement geometry.

provides more stable acceleration command and requires less energy consumption, especially when the cross coupling effect is strong.

The remainder of this Note is organized as follows. The problem formulation is described in Sec. III Section III presents the details of the proposed 3D impact time guidance law, followed by the property analysis shown in Sec. IV Finally, some simulation results and conclusions are offered.

\section{Problem Formulation}

This section states the problem formulation of this paper. Before introducing the system kinematics, we make three basic assumptions as follows:

Assumption 1 The target is stationary.

Assumption 2 The missile is assumed as an ideal point-mass model.

Assumption 3 The missile is flying with constant velocity.

Note that these assumptions are widely accepted in impact time guidance law design for anti-ship missiles: (Assumption 1) Compared to anti-ship missiles, their target' speed is ignorable. (Assumption 2) Typical philosophy treats the guidance and control loops separately by placing the guidance kinematics in an outer-loop, generating guidance commands tracked by an inner dynamic control loop, also known as autopilot. (Assumption 3) The vehicle's velocity is generally slowly varying and hence can be assumed as piece-wise constant. 
Under these assumptions, the 3D engagement geometry is shown in Fig. 1 , where $\left(X_{I}, Y_{I}, Z_{I}\right)$ denotes the inertial reference coordinate system and $V$ is the missile velocity. The missile-target relative range is denoted as $R$. The notations $\theta_{M}$ and $\phi_{M}$ stand for two velocity lead angles with respect to the LOS line in pitch and yaw planes, respectively. Note that both $\theta_{M}$ and $\phi_{M}$ can be obtained indirectly from the onboard seeker gimbal angles [26]. The variables $\theta_{L}$ and $\phi_{L}$ represent the LOS angles in azimuth and elevation directions, respectively. The angle $\sigma$ is missile velocity lead angle in the engagement plane, e.g., 'total' velocity lead angle, also known as heading error. The differential equations describing the 3D kinematics can be formulated as [26]

$$
\begin{gathered}
\dot{R}=-V \cos \theta_{M} \cos \phi_{M} \\
\dot{\theta}_{L}=-\frac{V}{R} \sin \theta_{M} \\
\dot{\phi}_{L}=-\frac{V}{R \cos \theta_{L}} \cos \theta_{M} \sin \phi_{M} \\
\dot{\theta}_{M}=\frac{a_{z}}{V}+\frac{V}{R} \cos \theta_{M} \sin ^{2} \phi_{M} \tan \theta_{L}+\frac{V}{R} \sin \theta_{M} \cos \phi_{M} \\
\dot{\phi}_{M}=\frac{a_{y}}{V \cos \theta_{M}}-\frac{V}{R} \sin \theta_{M} \sin _{M} \cos \phi_{M} \tan \theta_{L} \\
+\frac{V}{R \cos \theta_{M}} \sin ^{2} \theta_{M} \sin \phi_{M}+\frac{V}{R} \cos \theta_{M} \sin \phi_{M}
\end{gathered}
$$

where $a_{y}$ and $a_{z}$ are missile accelerations in yaw and pitch directions, respectively.

The complementary equation defining the relationship between the heading error and the projected velocity lead angles can be obtained from Fig. 1] as

$$
\cos \sigma=\cos \theta_{M} \cos \phi_{M}
$$

The aim of this Note is to design a 3D optimal guidance law such that the missile can intercept a stationary target with a specific impact time $t_{d}$. Our solution to this problem is given by a composite guidance command, consisting of an optimal baseline 3D PNG and an optimal impact time error feedback term.

\section{Three-Dimensional Optimal Impact Time Guidance Law Design and Analysis}

This section will present the details of the proposed impact time guidance law. We will first predict the impact time under 3D PNG and then design an error feedback term using optimal error dynamics.

\section{A. Impact Time Prediction in Three-Dimensional Engagement}

In impact time control, accurate impact time prediction is of paramount importance. For this reason, this subsection will generalize the 2D PNG-based time-to-go estimation [6, 23] to practical 3D scenarios. The classical PNG generates 
the commanded acceleration of the interceptor in proportion to the turning rate of LOS. In a 3D scenario, PNG is defined in a vector format as [26]

$$
\boldsymbol{a}^{\mathrm{PNG}}=N \boldsymbol{\Omega}_{L} \times \boldsymbol{V}
$$

where $N>0$ denotes the navigation gain. The notations $\boldsymbol{\Omega}_{L}$ and $\boldsymbol{V}$ represent the LOS angular rate and missile velocity vectors, respectively. These two vectors determine the engagement plane of the 3D interception geometry [27]. Since the relative range is usually not adjustable during terminal guidance phase, the 3D PNG is usually implemented in two planes in the velocity coordinate as [26]

$$
\begin{gathered}
a_{y}^{\mathrm{PNG}}=-N V \dot{\lambda}_{y} \sin \theta_{M} \sin \phi_{M}+N V \dot{\lambda}_{z} \cos \theta_{M} \\
a_{z}^{\mathrm{PNG}}=-N V \dot{\lambda}_{y} \cos \phi_{M}
\end{gathered}
$$

where $\dot{\lambda}_{y}$ and $\dot{\lambda}_{z}$ are LOS angular velocity vector components in the LOS coordinate, which can be directly measured using onboard seekers. Since

$$
\begin{gathered}
\dot{\lambda}_{y}=\frac{V}{R} \sin \theta_{M} \\
\dot{\lambda}_{z}=-\frac{V}{R} \cos \theta_{M} \sin \phi_{M}
\end{gathered}
$$

Substituting Eqs.(10)-(11) and (8)-(9) into it yields

$$
\begin{gathered}
a_{y}^{\mathrm{PNG}}=-\frac{N V^{2}}{R} \sin \phi_{M} \\
a_{z}^{\mathrm{PNG}}=-\frac{N V^{2}}{R} \sin \theta_{M} \cos \phi_{M}
\end{gathered}
$$

Differentiating Eq. (6) and substituting Eqs.(4)-(5) and Eqs. (12)-(13) into it results in

$$
\begin{aligned}
\dot{\sigma} & =\frac{1}{\sin \sigma}\left(\sin \theta_{M} \cos \phi_{M} \dot{\theta}_{M}+\cos \theta_{M} \sin \phi_{M} \dot{\phi}_{M}\right) \\
& =\frac{1}{\sin \sigma}\left[-\frac{(N-1) V}{R} \sin ^{2} \theta_{M} \cos ^{2} \phi_{M}-\frac{(N-1) V}{R} \sin ^{2} \phi_{M}\right] \\
& =-\frac{(N-1) V}{R \sin \sigma}\left(\cos ^{2} \phi_{M}-\cos ^{2} \theta_{M} \cos ^{2} \phi_{M}+\sin ^{2} \phi_{M}\right) \\
& =-\frac{(N-1) V}{R} \sin \sigma
\end{aligned}
$$

Dividing Eq. (1) by Eq. (14) yields

$$
\frac{d R}{d \sigma}=\frac{R \cot \sigma}{N-1}
$$


Solving differential equation 15 in terms of $\sigma$ gives

$$
R=\frac{R_{0}}{\left(\sin \sigma_{0}\right)^{1 /(N-1)}}(\sin \sigma)^{1 /(N-1)}
$$

where $R_{0}$ and $\sigma_{0}$ stand for the initial relative range and velocity lead angle.

Assume that the velocity leading angle satisfies $|\sigma|<\pi / 2$, which implies that $R$ is strictly decreasing from Eq. (1). Define an auxiliary variable $\eta=\sin \sigma$, then, Eq. (1) can be reformulated as

$$
\frac{d t}{d R}=-\frac{1}{V \sqrt{1-\eta^{2}}}
$$

Integrating the preceding expression using binomial series gives the predicted impact time $t_{f}$ as

$$
\begin{aligned}
t_{f} & =\frac{1}{V} \int_{0}^{R_{0}} \frac{1}{\sqrt{1-\eta^{2}}} d R \\
& =\frac{1}{V} \int_{0}^{R_{0}}\left(1+\frac{1}{2} \eta^{2}+\frac{3}{8} \eta^{4}+\frac{5}{16} \eta^{6}+\cdots\right) d R
\end{aligned}
$$

Substituting Eq. (16) into Eq. (18) and after integration, we have

$$
t_{f}=\frac{R_{0}}{V}\left[1+\frac{\eta_{0}^{2}}{2(2 N-1)}+\frac{3 \eta_{0}^{4}}{8(4 N-3)}+\frac{5 \eta_{0}^{6}}{16(6 N-5)}+\cdots\right]
$$

where $\eta_{0}$ denotes the initial value of $\eta$.

Replacing $R_{0}$ and $\eta_{0}$ with $R$ and $\eta$, respectively, gives the predicted time-to-go under 3D PNG as

$$
t_{g o}=\frac{R}{V}\left[1+\frac{\eta^{2}}{2(2 N-1)}+\frac{3 \eta^{4}}{8(4 N-3)}+\frac{5 \eta^{6}}{16(6 N-5)}+\cdots\right]
$$

By neglecting the higher order terms of $\eta^{2}$, we have the approximated time-to-go estimation as

$$
t_{g o}=\frac{R}{V}\left[1+\frac{\sin ^{2} \sigma}{2(2 N-1)}\right]
$$

Remark 1 For practical interceptors that provide roll stabilization capability, the 3D guidance problem can be treated in two separate channels. Accordingly, 3D homing guidance can also be achieved by constructing two separate $2 D$ PNGs in the pitch and yaw planes of the missile for roll-stabilized airframes. The commanded accelerations in the two planes are defined as [28]

$$
a_{y}^{P N G}=-\frac{N V^{2}}{R} \sin \phi_{M}
$$




$$
a_{z}^{P N G}=-\frac{N V^{2}}{R} \sin \theta_{M}
$$

It follows from Eqs. (12) and (13) that separate $2 D P N G$ is identical to $3 D P N G$ if the cross coupling between the pitch and the yaw planes is ignorable. However, if the relative motions in the two planes can not be decoupled, performance degradation of separately implementing $2 D P N G$ is inevitable due to the cross coupling effect.

Remark 2 If we only consider the $2 D$ engagement, pitch plane for example, we have $\sigma=\theta_{M}$ and $\phi_{M}=0$. Then, the predicted time-to-go 20 reduces to

$$
t_{g o}=\frac{R}{V}\left[1+\frac{\sin ^{2} \theta_{M}}{2(2 N-1)}\right]
$$

which coincides with the results, derived in [23], when the velocity leading angle is small, e.g., $\sin \theta_{M} \approx \theta_{M}$. Comparing Eqs. (20) and 24], it can be concluded that the proposed time-to-go estimation extends the $2 D$ algorithm to a projected plan containing the LOS vector and missile velocity vector in the $3 D$ scenario.

Remark 3 In impact time guidance law design, the desired impact time $t_{d}$ should be set to be achievable, e.g., the problem is well posed. From practical standpoint of view, the desired impact time $t_{d}$ is required to be larger than the predicted impact time $t_{f}$. For this reason, a suitable choice of $t_{d}$ is

$$
t_{d}>\frac{R_{0}}{V}\left[1+\frac{\sin ^{2} \sigma_{0}}{2(2 N-1)}\right]
$$

\section{B. Impact Time Guidance Law Design}

To achieve impact time control, both target interception and zero impact time error are required to be satisfied. For this reason, we propose a 3D composite guidance law, which is composed of an optimal baseline 3D PNG and an optimal impact time error feedback term. Instead of using two different biased terms, the proposed guidance law only utilizes one unique feedback command, which is automatically allocated to both vertical and horizontal planes, as

$$
\begin{gathered}
a_{y}=a_{y}^{\mathrm{PNG}}+a_{b} \sin \phi_{M}=\left(-\frac{N V^{2}}{R}+a_{b}\right) \sin \phi_{M} \\
a_{z}=a_{z}^{\mathrm{PNG}}+a_{b} \sin \theta_{M} \cos \phi_{M}=\left(-\frac{N V^{2}}{R}+a_{b}\right) \sin \theta_{M} \cos \phi_{M}
\end{gathered}
$$

where $a_{b}$ denotes the error feedback term to be determined.

Define $e_{t}=t_{d}-t_{g o}-t$ as the impact time error. Substituting 21] into $e_{t}$ and taking its time derivative using Eqs. 
(26) and (27) gives

$$
\begin{aligned}
\dot{e}_{t} & =-\dot{t}_{g o}-1 \\
& =-\frac{\dot{R}}{V}-\frac{\dot{R}}{V} \frac{\sin ^{2} \sigma}{2(2 N-1)}-\frac{R}{V} \frac{\sin \sigma \cos \sigma \dot{\sigma}}{2 N-1}-1 \\
& =\cos \sigma\left[1+\frac{\sin ^{2} \sigma}{2(2 N-1)}\right]-\frac{R \cos \sigma}{(2 N-1) V^{2}}\left[-\frac{(N-1) V^{2}}{R} \sin ^{2} \sigma+a_{b} \sin ^{2} \sigma\right]-1 \\
& =\cos \sigma\left[1+\frac{\sin ^{2} \sigma}{2(2 N-1)}\right]-\frac{R \cos \sigma \sin ^{2} \sigma}{(2 N-1) V^{2}}\left[-\frac{(N-1) V^{2}}{R}+a_{b}\right]-1 \\
& =\cos \sigma\left[1+\frac{\sin ^{2} \sigma}{2(2 N-1)}\right]+\frac{(N-1) \cos \sigma \sin ^{2} \sigma}{2 N-1}-\frac{R \cos \sigma \sin ^{2} \sigma}{(2 N-1) V^{2}} a_{b}-1
\end{aligned}
$$

Assume that the missile velocity lead angle $\sigma$ is small. Then, we have $\sin \sigma \approx \sigma$ and $\cos \sigma \approx 1-\sigma^{2} / 2$. Using these two approximations and neglecting higher order terms of $\sigma$, Eq. 28 reduces to

$$
\dot{e}_{t}=-\frac{R \sin ^{2} \sigma}{(2 N-1) V^{2}} a_{b}
$$

For system [29], consider the following optimal error dynamics [8]

$$
\dot{e}_{t}+\frac{K}{t_{g o}} e_{t}=0
$$

where $K>0$ is the guidance gain to be designed.

Combining Eq. 29] with Eq. (30) gives the guidance command to nullify the impact time error as

$$
a_{b}=\frac{K(2 N-1) V^{2}}{R \sin ^{2} \sigma t_{g o}} e_{t}
$$

Substituting Eq. (31) into Eqs. 26-27) yields the explicit guidance command as

$$
\begin{gathered}
a_{y}=\left[-\frac{N V^{2}}{R}+\frac{K(2 N-1) V^{2}}{R \sin ^{2} \sigma t_{g o}} e_{t}\right] \sin \phi_{M} \\
a_{z}=\left[-\frac{N V^{2}}{R}+\frac{K(2 N-1) V^{2}}{R \sin ^{2} \sigma t_{g o}} e_{t}\right] \sin \theta_{M} \cos \phi_{M}
\end{gathered}
$$

Similar 3D PNG, the proposed guidance law can be formulated as a vector, locating in the engagement plane, as

$$
\boldsymbol{a}=\left[-\frac{N V^{2}}{R} \sin \sigma+\frac{K(2 N-1) V^{2}}{R \sin \sigma t_{g o}} e_{t}\right] \boldsymbol{e}_{a}
$$

where $\boldsymbol{e}_{a}=\left[0, \sin \phi_{M} / \sin \sigma, \sin \theta_{M} \cos \phi_{M} / \sin \sigma\right]^{T}$ denotes the unit vector that specifies the direction of the commanded 
acceleration in the velocity coordinate.

Remark 4 Although the proposed 3D guidance law is derived using stationary targets, the guidance law developed can be easily adapted to non-maneuvering target scenarios through the well-known predicted interception point concept $[15]$.

\section{Analysis of the Proposed Guidance Law}

This section analyzes the properties of the proposed 3D optimal impact time guidance law in the following aspects.

\section{A. Singularity Issue}

From Eq. (31), we can observe that $\sigma=0$ is a singular point, which will result in infinite guidance command. However, it is easy to verify that this singular point is trivial since the velocity lead angle $\sigma \neq 0$ except for the final impact point. To see this, taking the time derivative of $\sigma$ and substituting Eqs. (32)-33) into it yields

$$
\dot{\sigma}=-\frac{(N-1) V}{R} \sin \sigma+\frac{K(2 N-1) V}{R \sin \sigma t_{g o}} e_{t}
$$

By choosing the desired impact time $t_{d}$ that satisfies condition 25 , we can readily conclude that $e_{t, 0}>0$, where $e_{t, 0}$ denotes the initial impact time error. With this in mind, one can imply that the term $K(2 N-1) V e_{t} /\left(R \sin \sigma t_{g o}\right)$ initially tries to increase the magnitude of the velocity lead angle for reducing the impact time error. Also note that the PNG term $-(N-1) V \sin \sigma / R$ is utilized to regulate the velocity lead angle to zero to guarantee target interception. It is well known that the velocity lead angle under PNG converges to zero only at the time of impact [1]. Therefore, if the guidance gain $K$ satisfies

$$
\frac{K(2 N-1) V}{R_{0}\left|\sin \sigma_{0}\right| t_{f}} e_{t, 0}>\frac{(N-1) V}{R_{0}}\left|\sin \sigma_{0}\right|
$$

or the equivalent form

$$
K>\frac{(N-1) \sin ^{2} \sigma_{0} t_{f}}{(2 N-1) e_{t, 0}}
$$

the error feedback term $a_{b}$ will play a dominant role initially in the guidance command, thus forcing the magnitude of the velocity lead angle increases until certain time instant $t_{1}$. When $t \geq t_{1}$, the PNG term will dominate over $a_{b}$, hence regulating the magnitude of the velocity lead angle to zero at the time of impact. Note that condition (37) is easily to be satisfied for practical scenarios. Therefore, the proposed guidance law is nonsingular. An example of the velocity leading angle response under the proposed guidance law is presented in Fig. 2. 


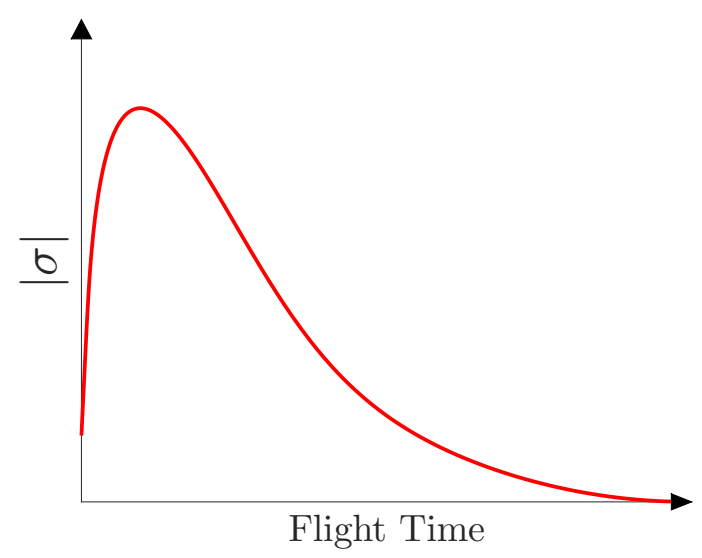

Fig. 2 An example of velocity leading angle profile.

\section{B. Finite-Time Convergence of Impact Time Error}

Under optimal error dynamics [30, it is easy to verify that the closed-form solution of the impact time error is determined as

$$
e_{t}=e_{t, 0}\left(\frac{t_{g o}}{t_{f}}\right)^{K}
$$

which clearly reveals that the impact time error $e_{t}$ will converge to zero at the time of impact if $K>0$, thus satisfying the impact time control requirement. Furthermore, the convergence rate of impact time error is determined by the guidance gain $K$ : larger $K$ results in faster convergence since $t_{g o} / t_{f} \leq 1$.

\section{Optimality of the Error Feedback Term}

According to Theorem 1 in [8], error dynamics (30) is optimal in terms of performance index

$$
J=\frac{1}{2} \int_{t}^{t_{f}} \frac{R^{2} \sin ^{4} \sigma}{(2 N-1)^{2} V^{4}\left(t_{f}-\tau\right)^{K-1}} a_{b}(\tau) d \tau
$$

Since the constant terms in the performance index do not affect the optimal pattern, the previously mentioned performance index is identical to

$$
J=\frac{1}{2} \int_{t}^{t_{f}} \frac{R^{2} \sin ^{4} \sigma}{\left(t_{f}-\tau\right)^{K-1}} a_{b}(\tau) d \tau
$$

It follows from Eq. (40) that the weighting function $R^{2} \sin ^{4} \sigma /\left(t_{f}-\tau\right)^{K-1}$ gradually decreases with the decrease of $R$ and $\sigma$. This means that the magnitude of the error feedback term $a_{b}$ tends to increase when the missile approaches the target. This property is, obviously, not desirable to guarantee finite guidance command. For this reason, it is recommended to choose relatively large guidance gain $K$ such that $t_{g o}^{K-1}$ is larger than $R^{2} \sin ^{4} \sigma$ to compensate for the decreasing of the weighting function. Notice that the decreasing rates of both relative range and velocity lead angle are 
governed by the PNG term, a suitable choice of $K$ is $K>N$.

\section{Relationship with 2D Optimal Impact Time Guidance Law [8]}

When only considering the 2D homing engagement, e.g., pitch plan for example, we have $\sigma=\theta_{M}$ and $\phi_{M}=0$. Then, the proposed 3D impact time guidance law, shown in Eqs. 32. 33, reduces to

$$
\begin{gathered}
a_{y}=0 \\
a_{z}=-\frac{N V^{2}}{R} \sin \theta_{M}+\frac{K(2 N-1) V^{2}}{R \sin \theta_{M} t_{g o}} e_{t}
\end{gathered}
$$

which coincides with the generalized 2D optimal impact time guidance law proposed in [8].

It is well-known that the 2D guidance law can be directly applied to 3D scenarios for roll-stabilized airframes by ignoring the cross-coupling effect between the horizontal and the vertical channels, e.g., assuming $\theta_{M}$ and $\phi_{M}$ are small. Under the condition that the relative motions in the two planes are decoupled, impact time control in a 3D scenario can be satisfied by using separate $2 \mathrm{D}$ guidance laws in the two planes. One feasible strategy to achieve this objective is to apply the $2 \mathrm{D}$ optimal impact time guidance law [8] in the vertical plane for impact time control and utilize the 2D PNG in the horizontal plane for the homing constraint. With this in mind, the individual 2D guidance command can be obtained as

$$
\begin{gathered}
a_{y}^{2 \mathrm{D}}=-\frac{N V^{2}}{R} \sin \phi_{M} \\
a_{z}^{2 \mathrm{D}}=-\frac{N V^{2}}{R} \sin \theta_{M}+\frac{K(2 N-1) V^{2}}{R \sin \theta_{M} t_{g o}} e_{t}
\end{gathered}
$$

Comparing Eqs. (32)-33 with Eqs. (43)-(44), one can observe that the proposed 3D guidance law automatically distributes the error feedback command term to both horizontal and vertical plans while the 2D guidance law only leverages one channel in impact time control. This means that the proposed 3D guidance law fully exploits the the synergism between these two channels and thus is beneficial when $\theta_{M} \neq 0$ and $\phi_{M} \neq 0$, especially when the effect of cross coupling is strong. For example, if $\sin \theta_{M} \cos \phi_{M}>\sin \phi_{M}$, the proposed guidance law will mainly utilize the vertical plane for impact time control. Similarly, if $\sin \phi_{M}$ is dominant over $\sin \theta_{M} \cos \phi_{M}$, the horizontal plane will play an important role in impact time control. This property will be empirically evaluated in simulations. It is worthy pointing out that the performance of 3D impact time guidance law is close to its $2 \mathrm{D}$ counterpart only when $\phi_{M}$ is small. As separately implementing 2D guidance law ignores the cross coupling effect, performance degradation is inevitable if this small angle approximation. For example, if $\theta_{M}$ approaches to near zero before interception, the pitch guidance command (44) will suffer from a singular issue, as can be observed from the simulation studies.

Notice that the proposed 3D guidance leverages a kind of automatic command allocation, it is, therefore, helpful in 
saving energy consumption, compared to the separate 2D guidance law. To see this, define $E=a_{y}^{2}+a_{z}^{2}$ as the quadratic energy consumption at each time instant, then the required energy of the proposed 3D guidance law can be obtained as

$$
\begin{aligned}
E_{3 \mathrm{D}} & =\left[-\frac{N V^{2}}{R}+\frac{K(2 N-1) V^{2}}{R \sin ^{2} \sigma t_{g o}} e_{t}\right]^{2}\left(\sin ^{2} \phi_{M}+\sin ^{2} \theta_{M} \cos ^{2} \phi_{M}\right) \\
& =\left[-\frac{N V^{2}}{R}+\frac{K(2 N-1) V^{2}}{R \sin ^{2} \sigma t_{g o}} e_{t}\right]^{2} \sin ^{2} \sigma \\
& =\left(\frac{N^{2} V^{4}}{R^{2}}\right) \sin ^{2} \sigma-\frac{2 K N(2 N-1) V^{4} e_{t}}{R^{2} t_{g o}}+\frac{K^{2}(2 N-1)^{2} V^{4}}{R^{2} \sin ^{2} \sigma t_{g o}^{2}} e_{t}^{2}
\end{aligned}
$$

The required energy of utilizing separate $2 \mathrm{D}$ guidance law is given by

$$
\begin{aligned}
E_{2 \mathrm{D}} & =\left(-\frac{N V^{2}}{R} \sin \phi_{M}\right)^{2}+\left(-\frac{N V^{2}}{R} \sin \theta_{M}+\frac{K(2 N-1) V^{2}}{R \sin \theta_{M} t_{g o}} e_{t}\right)^{2} \\
& =\left(\frac{N^{2} V^{4}}{R^{2}}\right)\left(\sin ^{2} \phi_{M}+\sin ^{2} \theta_{M}\right)-\frac{2 K N(2 N-1) V^{4} e_{t}}{R^{2} t_{g o}}+\frac{K^{2}(2 N-1)^{2} V^{4}}{R^{2} \sin ^{2} \theta_{M} t_{g o}^{2}} e_{t}^{2}
\end{aligned}
$$

Since $\cos ^{2} \sigma=\cos ^{2} \theta_{M} \cos ^{2} \phi_{M} \leq \cos ^{2} \theta_{M}$, we have $\sin ^{2} \sigma \geq \sin ^{2} \theta_{M}$. Then, it follows from Eq. (47) that

$$
\begin{aligned}
E_{2 \mathrm{D}} & \geq\left(\frac{N^{2} V^{4}}{R^{2}}\right)\left(\sin ^{2} \phi_{M}+\sin ^{2} \theta_{M} \cos ^{2} \phi_{M}\right)-\frac{2 K N(2 N-1) V^{4} e_{t}}{R^{2} t_{g o}}+\frac{K^{2}(2 N-1)^{2} V^{4}}{R^{2} \sin ^{2} \sigma t_{g o}^{2}} e_{t}^{2} \\
& =\left(\frac{N^{2} V^{4}}{R^{2}}\right) \sin ^{2} \sigma-\frac{2 K N(2 N-1) V^{4} e_{t}}{R^{2} t_{g o}}+\frac{K^{2}(2 N-1)^{2} V^{4}}{R^{2} \sin ^{2} \sigma t_{g o}^{2}} e_{t}^{2} \\
& =E_{3 \mathrm{D}}
\end{aligned}
$$

where the equality holds if and only if $\phi_{M}=0$. This expression clearly shows that the proposed 3D guidance law requires less energy consumption than separately implementing the $2 \mathrm{D}$ guidance law.

\section{Numerical Simulations}

In this section, the effectiveness of the proposed 3D optimal impact time guidance law is demonstrated through numerical simulations, in which an anti-ship missile is considered to intercept a stationary target. In the considered scenario, the target is located at $(0 \mathrm{~m}, 0 \mathrm{~m}, 0 \mathrm{~m})$. The interceptor initially locates at $(6000 \mathrm{~m}, 6000 \mathrm{~m}, 0 \mathrm{~m})$ with initial velocity lead angles $\theta_{M, 0}=10^{\circ}$ and $\phi_{M, 0}=10^{\circ}$. The missile flies with constant velocity $V=250 \mathrm{~m} / \mathrm{s}$. For implementing the proposed guidance law, the navigation gain of the baseline PNG is set as $N=3$. In practice, the achieved acceleration of the missile is always bounded due to physical limits. For this reason, the magnitudes of both $a_{y}$ and $a_{z}$ are constrained by $100 m / s^{2}$ in simulations. 


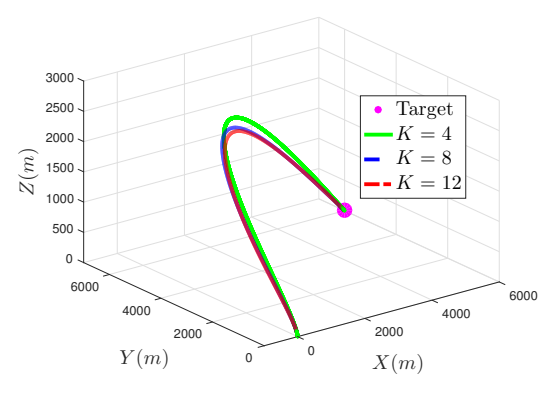

(a) Interception trajectories

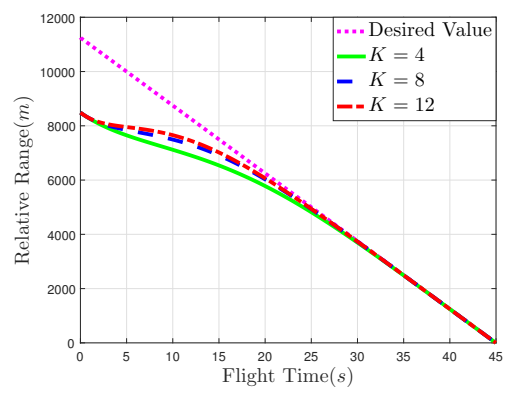

(b) Relative range

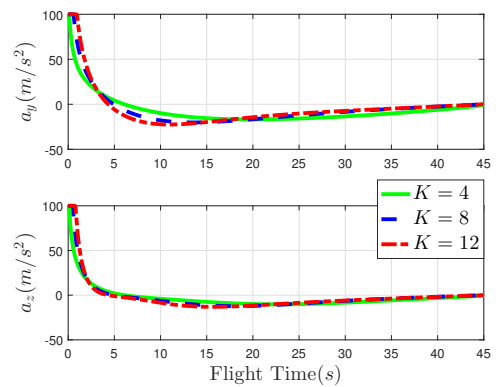

(c) Acceleration command

Fig. 3 Simulation results with respect to different guidance gains $K$.

\section{A. Characteristics of the Proposed Three-Dimensional Impact Time Guidance Law}

This subsection will empirically analyze the properties of the proposed 3D optimal impact time guidance law under various conditions. It is clear that the guidance gain $K$ plays an important role in the proposed guidance law since it governs the convergence rate of the impact time error. For this reason, we first perform simulations with various guidance gains $K=4,8,12$. In these simulations, the desired impact time is set as $t_{d}=45 \mathrm{~s}$, which satisfies condition 25. The simulation results, including interception trajectories, history of relative range and acceleration command, are presented in Fig. 3 From this figure, it is clear that the proposed 3D guidance law successfully drives the missile to intercept the stationary target with desired impact time. With higher guidance gain $K$, the response pattern of the relative range becomes more curved, thus closer to the desired pattern $\left(t_{d}-t\right) V$. That is, higher guidance gain $K$ helps to increase the convergence speed of the impact time error. However, Fig. 3 (c) reveals that the proposed guidance law with higher guidance gain $K$ requires more control energy during the initial flight phase. Furthermore, by choosing $K>N$, we can clearly note from Fig. 3 (d) that the guidance commands in both vertical and horizontal planes converge to zero at the time of impact, demonstrating that the proposed guidance law has enough operational margins to cope with undesired disturbances when the missile approaches the target.

Now, let us investigate the performance of the proposed 3D impact time guidance law with respect to different desired impact time $t_{d}=40 \mathrm{~s}, 60 \mathrm{~s}, 80 \mathrm{~s}$. For implementing the proposed guidance law, the guidance gain of the impact time error feedback term is chosen as $K=12$. Fig. 4 (a) compares the interceptor trajectory for these three different impact time constraints. This figure clearly shows that the interceptor takes a longer flight path with a larger desired impact time. The history of the relative range with the three cases of desired impact time $t_{d}$ is presented in Fig. 4 (b), which reveals that the proposed guidance law satisfies the impact time constraint precisely. The impact time error of the proposed guidance law turns out to be less than $0.01 s$ with different impact time constraints in our simulations. From this figure, it is obviously that longer convergence phase is required to regulate the impact time error with larger desired impact time $t_{d}$ under the same initial conditions. The missile acceleration command produced by the proposed guidance 


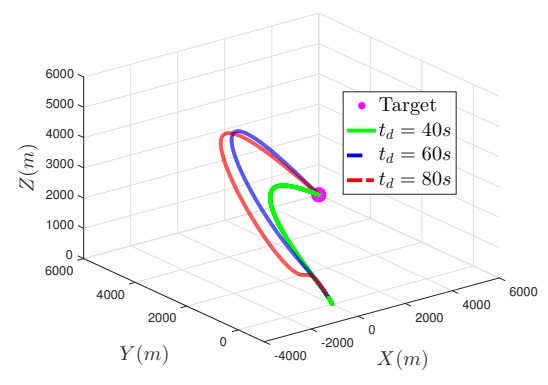

(a) Interception trajectories

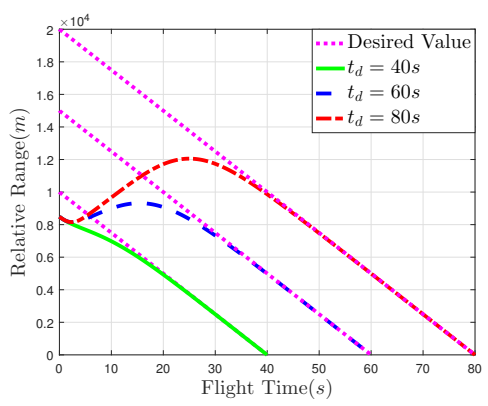

(b) Relative range

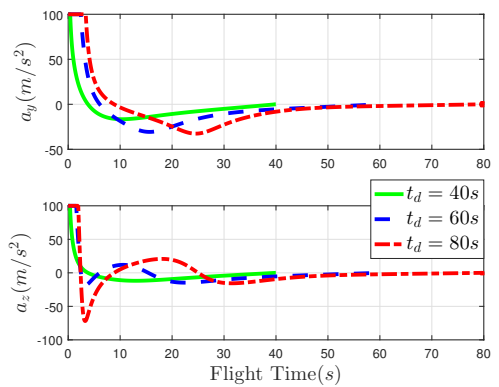

(c) Acceleration command

Fig. 4 Simulation results with respect to different desired impact time $t_{d}$.

law with different $t_{d}$ is provided in Fig. 4(c). Clearly, more energy consumption is required during the initial phase for a larger $t_{d}$. For this reason, the duration of initial acceleration saturation of $t_{d}=80 \mathrm{~s}$ is longer than that of $t_{d}=40 \mathrm{~s}$ and $t_{d}=60 s$.

\section{B. Comparison with Two-Dimensional Impact Time Guidance Law [8]}

To further show the advantages of the proposed 3D impact time guidance law, comparisons with generalized 2D optimal impact time guidance law [8] are conducted in this subsection. For the purpose of comparison, three different initial conditions are considered as: (1) $\theta_{M, 0}=40^{\circ}, \phi_{M, 0}=0^{\circ}$; (2) $\theta_{M, 0}=40^{\circ}, \phi_{M, 0}=40^{\circ}$; and $\theta_{M, 0}=40^{\circ}$, $\phi_{M, 0}=80^{\circ}$. It is clear that case 1 is a $2 \mathrm{D}$ homing scenario occurs in the vertical plane; case 2 considers moderate level of coupling effect between two planes; and case 3 corresponds to the strong cross coupling scenario. For fair comparison, the guidance gain of the impact time error feedback term for both guidance laws is set as $K=12$.

The simulation results, including interception trajectories, relative range and acceleration command, are shown in Fig. 5 with desired impact time $t_{d}=45 \mathrm{~s}$. From the first row of Fig. 5 , one can observe that these two guidance laws generate exactly the same results for case 1, as we expected. The reason is that the proposed 3D impact time guidance law reduces its $2 \mathrm{D}$ counterpart if we only consider the vertical plane as we discussed before. For case 2 , since $\sin \theta_{M} \cos \phi_{M}<\sin \phi_{M}$, the proposed 3D guidance law will mainly utilize the horizontal plane to regulate the impact time error, as shown in the second row of Fig. 5. As we utilize the same guidance gain $K$ for both guidance laws, the impact time error dynamics under the two guidance laws is the same. For this reason, the convergence patterns of the relative range under both guidance laws show similar characteristics, as can be confirmed from Fig. 5 (e). From Fig. 5(f), we can observe that the proposed 3D guidance law leverages both vertical and horizontal channels for impact and automatically distribute the error feedback command into these two channels. As a comparison, the 2D impact time guidance law only utilizes the vertical plane in impact time control. As for the strong cross coupling engagement scenario of case 3, both guidance laws successfully drive the missile to intercept the target with desired impact time constraint, as shown in the third row of Fig. 5. However, the 2D impact time guidance law shows oscillating patterns 


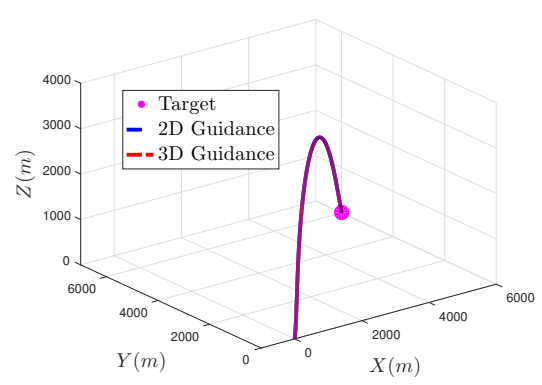

(a) Interception trajectories for case 1

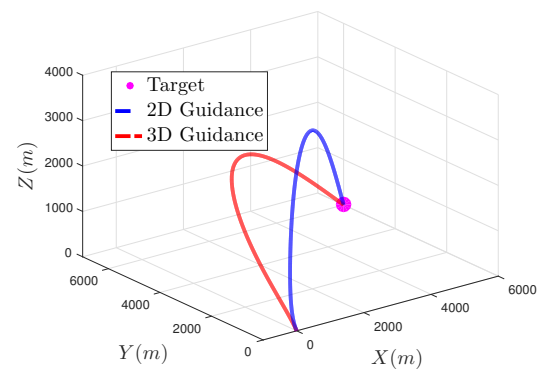

(d) Interception trajectories for case 2

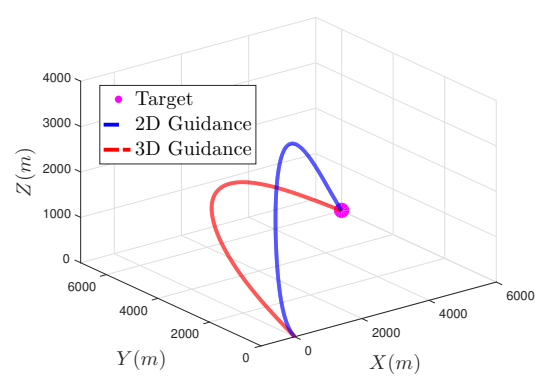

(g) Interception trajectories for case 3

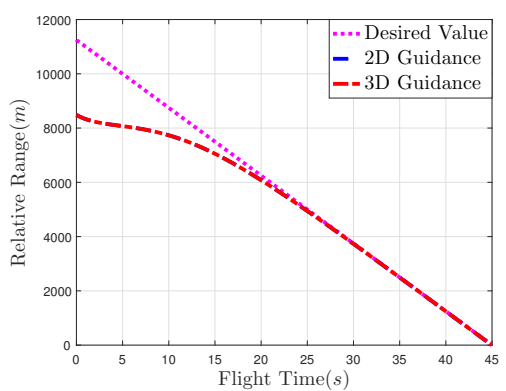

(b) Relative range for case 1

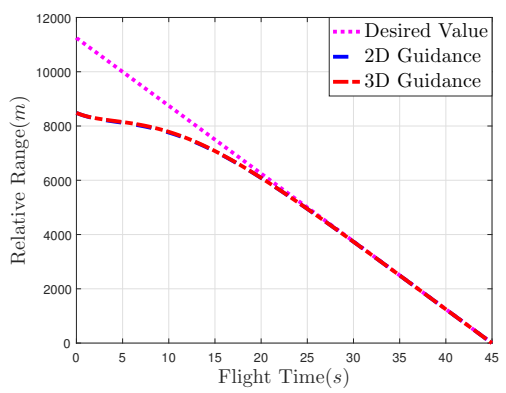

(e) Relative range for case 2

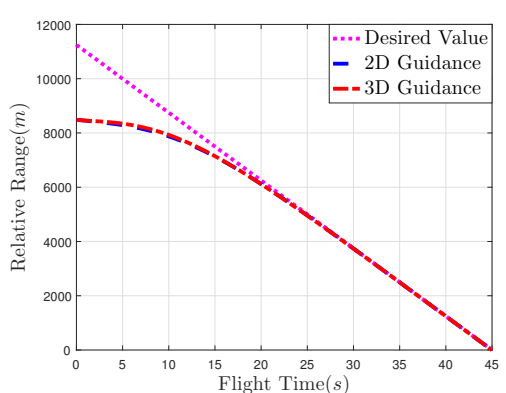

(h) Relative range for case 3
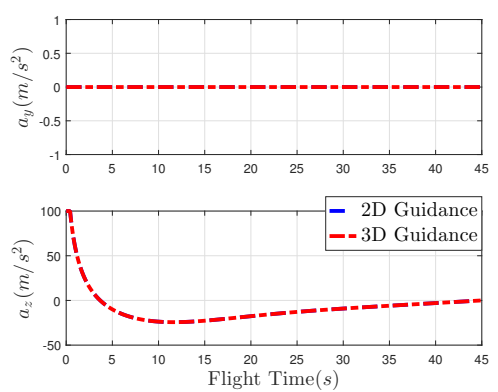

(c) Acceleration command for case 1
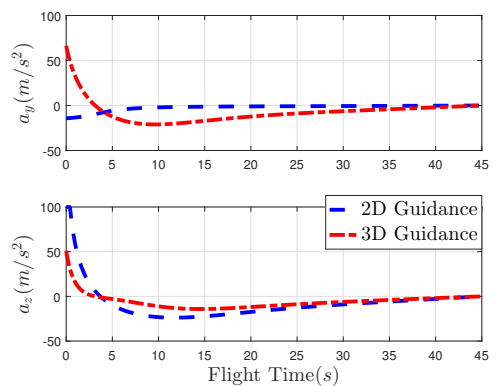

(f) Acceleration command for case 2

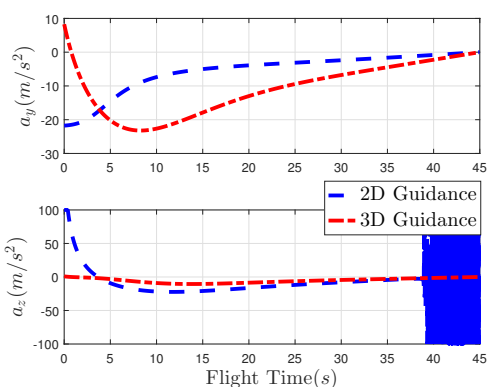

(i) Acceleration command for case 3

Fig. 5 Comparison results with 2D optimal impact time guidance law. 
Table 1 Control effort comparisons.

\begin{tabular}{l|ccc}
\hline \hline & Case 1 & Case 2 & Case 3 \\
\hline 2D Guidance & 18733 & 18951 & 62803 \\
\hline 3D Guidance & 18733 & 13080 & 9926 \\
\hline \hline
\end{tabular}

when the interceptor is close to the target, which is not desirable for onboard control systems. The control effort $\int_{t_{0}}^{t_{f}}\left[a_{y}^{2}(t)+a_{z}^{2}(t)\right] d t$ obtained from both 2D and 3D impact time guidance laws for these three different cases are summarized in Table 1. From this table, we can readily note that the proposed 3D impact guidance law helps to reduce the energy consumption except for the particular case 1. This confirms the theoretical findings presented in the previous section.

\section{Conclusions}

The homing guidance problem for impact time control against a stationary target in 3D scenarios is discussed in this Note. The proposed optimal guidance law is composed of two different parts: an baseline 3D PNG and an impact time error feedback error. The baseline PNG is utilized for target interception while the feedback command regulates the impact time error to zero in finite time. To determine the error feedback term, we also generalize the original 2D time-to-go estimation algorithm to the realistic 3D engagement. Compared to the 2D generalized optimal impact time guidance law [8], the proposed approach is proved to be helpful in energy saving and fully exploits the the synergism between the horizontal and the vertical channels.

\section{References}

[1] Zarchan, P., Tactical and strategic missile guidance, American Institute of Aeronautics and Astronautics, 2012.

[2] Jeon, I.-S., and Lee, J.-I., “Optimality of proportional navigation based on nonlinear formulation,” IEEE Transactions on Aerospace and Electronic Systems, Vol. 46, No. 4, 2010, pp. 2051-2055. doi:10.1109/TAES.2010.5595614.

[3] Cho, N., and Kim, Y., "Optimality of augmented ideal proportional navigation for maneuvering target interception,” IEEE Transactions on Aerospace and Electronic Systems, Vol. 52, No. 2, 2016, pp. 948-954. doi:10.1109/TAES.2015.140432.

[4] Jeon, I.-S., Lee, J.-I., and Tahk, M.-J., "Impact-time-control guidance law for anti-ship missiles," IEEE Transactions on Control Systems Technology, Vol. 14, No. 2, 2006, pp. 260-266. doi:10.1109/TCST.2005.863655.

[5] Lee, J.-I., Jeon, I.-S., and Tahk, M.-J., "Guidance law to control impact time and angle," IEEE Transactions on Aerospace and Electronic Systems, Vol. 43, No. 1, 2007, pp. 301-310. doi:10.1109/TAES.2007.357135.

[6] Jeon, I.-S., Lee, J.-I., and Tahk, M.-J., "Impact-Time-Control Guidance with Generalized Proportional Navigation Based 
on Nonlinear Formulation," Journal of Guidance, Control, and Dynamics, Vol. 39, No. 8, 2016, pp. 1887-1892. doi: 10.2514/1.G001681.

[7] Cho, N., and Kim, Y., "Modified pure proportional navigation guidance law for impact time control," Journal of Guidance, Control, and Dynamics, Vol. 39, No. 4, 2016, pp. 852-872. doi:10.2514/1.G001618.

[8] He, S., and Lee, C.-H., "Optimality of Error Dynamics in Missile Guidance Problems,” Journal of Guidance, Control, and Dynamics, Vol. 41, No. 7, 2018, pp. 1620-1629. doi:10.2514/1.G003343.

[9] Wang, J., and Zhang, R., “Terminal Guidance for a Hypersonic Vehicle with Impact Time Control,” Journal of Guidance, Control, and Dynamics. doi:10.2514/1.G003540.

[10] Kim, M., Jung, B., Han, B., Lee, S., and Kim, Y., "Lyapunov-based impact time control guidance laws against stationary targets," IEEE Transactions on Aerospace and Electronic Systems, Vol. 51, No. 2, 2015, pp. 1111-1122. doi:10.1109/TAES.2014.130717.

[11] Saleem, A., and Ratnoo, A., "Lyapunov-based guidance law for impact time control and simultaneous arrival," Journal of Guidance, Control, and Dynamics, Vol. 39, No. 1, 2016, pp. 164-173. doi:10.2514/1.G001349.

[12] Chen, X., and Wang, J., "Nonsingular Sliding-Mode Control for Field-of-View Constrained Impact Time Guidance," Journal of Guidance, Control, and Dynamics, Vol. 41, No. 5, 2017, pp. 1214-1222. doi:10.2514/1.G003146.

[13] Kumar, S. R., and Ghose, D., "Sliding mode control based guidance law with impact time constraints," American Control Conference (ACC), 2013, IEEE, 2013, pp. 5760-5765. doi:10.1109/ACC.2013.6580740.

[14] Kumar, S. R., and Ghose, D., "Impact time guidance for large heading errors using sliding mode control," IEEE Transactions on Aerospace and Electronic Systems, Vol. 51, No. 4, 2015, pp. 3123-3138. doi:10.1109/TAES.2015.140137.

[15] Cho, D., Kim, H. J., and Tahk, M.-J., "Nonsingular sliding mode guidance for impact time control," Journal of Guidance, Control, and Dynamics, Vol. 39, No. 1, 2016, pp. 61-68. doi:10.2514/1.G001167.

[16] Harl, N., and Balakrishnan, S., "Impact time and angle guidance with sliding mode control," IEEE Transactions on Control Systems Technology, Vol. 20, No. 6, 2012, pp. 1436-1449. doi:10.1109/TCST.2011.2169795.

[17] Tekin, R., Erer, K. S., and Holzapfel, F., "Control of Impact Time with Increased Robustness via Feedback Linearization," Journal of Guidance, Control, and Dynamics, Vol. 39, No. 7, 2016, pp. 1682-1689. doi:10.2514/1.G001719.

[18] Tekin, R., Erer, K. S., and Holzapfel, F., "Polynomial Shaping of the Look Angle for Impact-Time Control," Journal of Guidance, Control, and Dynamics, Vol. 40, No. 10, 2017, pp. 2668-2673. doi:10.2514/1.G002751.

[19] Jeon, I.-S., and Lee, J.-I., "Impact-Time-Control Guidance Law with Constraints on Seeker Look Angle," IEEE Transactions on Aerospace and Electronic Systems, Vol. 53, No. 5, 2017, pp. 2621-2627. doi:10.1109/TAES.2017.2698837.

[20] Tekin, R., Erer, K. S., and Holzapfel, F., “Adaptive Impact Time Control via Look-Angle Shaping Under Varying Velocity,” Journal of Guidance, Control, and Dynamics, Vol. 40, No. 12, 2017, pp. 3247-3255. 
[21] Hu, Q., Han, T., and Xin, M., "New Impact Time and Angle Guidance Strategy via Virtual Target Approach,” Journal of Guidance, Control, and Dynamics. doi:10.2514/1.G003436.

[22] Livermore, R., Tsalik, R., and Shima, T., "Elliptic Guidance," Journal of Guidance, Control, and Dynamics. doi:10.2514/1. G003565.

[23] Jeon, I.-S., Lee, J.-I., and Tahk, M.-J., "Homing guidance law for cooperative attack of multiple missiles," Journal of Guidance, Control, and Dynamics, Vol. 33, No. 1, 2010, pp. 275-280. doi:10.2514/1.40136.

[24] Shiyu, Z., and Rui, Z., "Cooperative guidance for multimissile salvo attack,” Chinese Journal of Aeronautics, Vol. 21, No. 6, 2008, pp. 533-539. doi:10.1016/S1000-9361(08)60171-5.

[25] He, S., Wang, W., Lin, D., and Lei, H., “Consensus-Based Two-Stage Salvo Attack Guidance,” IEEE Transactions on Aerospace and Electronic Systems, Vol. 54, No. 3, 2018, pp. 1555-1566. doi:10.1109/TAES.2017.2773272.

[26] Song, S.-H., and Ha, I.-J., "A Lyapunov-like approach to performance analysis of 3-dimensional pure PNG laws,” IEEE Transactions on Aerospace and Electronic Systems, Vol. 30, No. 1, 1994, pp. 238-248. doi:10.1109/7.250424.

[27] Shin, H.-S., Tsourdos, A., and Li, K.-B., "A new three-dimensional sliding mode guidance law variation with finite time convergence," IEEE Transactions on Aerospace and Electronic Systems, Vol. 53, No. 5, 2017, pp. 2221-2232. doi: 10.1109/TAES.2017.2689938.

[28] Zhou, D., Sun, S., and Teo, K. L., "Guidance laws with finite time convergence," Journal of Guidance, Control, and Dynamics, Vol. 32, No. 6, 2009, pp. 1838-1846. doi:10.2514/1.42976. 
2018-11-21

Three-dimensional optimal impact time guidance for antiship missiles

He, Shaoming

AIAA

Shaoming He and Defu Lin. Three-dimensional optimal impact time guidance for antiship missiles. Journal of Guidance, Control, and Dynamics, Volume 42, Issue 4, 2019, pp. 941-948 https://doi.org/10.2514/1.G003971

Downloaded from Cranfield Library Services E-Repository 